Ann. Zootech., I973, 22 (I), 73-8I.

\title{
RÉACTION A L'ÉLECTRONARCOSE ET GLYCOGÉNOLYSE POST MORTEM CHEZ LE PORC
}

\author{
G. MONIN \\ avec la collaboration technique de A. Talmant et P. Vernin \\ Station de Recherches sur la Viande, \\ Centre de Recherches de Clermont-Ferrand, I. N. R. A., \\ Saint Genès Champanelle, 63110 Beaumont
}

\section{RÉSUMÉ}

11 est bien établi que la technique utilisée pour l'anesthésie des Porcs au cours de l'abattage peut influencer la vitesse de glycogénolyse musculaire post mortem et par suite la qualité de la viande. La présente étude a pour but de déterminer par quels mécanismes l'électronarcose est susceptible d'accélérer la chute du pH musculaire après l'abattage.

La température et quelques intermédiaires glycolytiques (ATP, G-6-P, F-I,6-P, acide lactique) étaient déterminés dans le muscle Longissimus dorsi de 17 porcs avant et après application d'un électrochoc (300 $V$, 50 périodes, I seconde), puis immédiatement après l'abattage. L'électrochoc entraîne une élévation de température et I'accumulation de G-6-P (probablement par activation de la phosphorylase). Il semble que du point de vue de la vitesse de chute du $\mathrm{pH}$ post mortem, l'augmentation de température due à l'électrochoc joue un rôle plus important que les perturbations de la glycolyse.

Les variations dans la durée d'application de l'électrochoc n'ont pas d'influence sur la glycogénolyse post mortem et la qualité de la viande. Les différences individuelles enregistrées en ce qui concerne ces caractéristiques paraissent devoir être attribuées à des différences de sensibilité des animaux à l'électrochoc, plutôt qu'à des variations dans le mode d'application de ce dernier. Le problème essentiel consiste donc à élucider plus complètement les mécanismes d'action de l'électronarcose au niveau du muscle et les causes physiologiques des différences de sensibilité entrc animaux.

\section{INTRODUCTION}

De nombreux travaux ont montré que la technique utilisée pour l'anesthésie des porcs lors de l'abattage était susceptible d'avoir de profondes répercussions sur la rapidité de la glycogénolyse post mortem. Ainsi la chute du $\mathrm{pH}$ musculaire après la mort est plus rapide chez les animaux saignés sous électronarcose que chez les animaux abattus sans étourdissement préalable (Mc LoughLIN, I97I ; RATCLIFF, 
I97I). Cet effet est imputable en partie aux contractions musculaires violentes engendrées par le passage du courant électrique dans les centres nerveux (BENDALl, I965; Mc LoUghlin et TARRANT, I968; SAIR et al., I970; MoniN, I97 I). Toutefois les conséquences propres de l'électronarcose sur les phénomènes biochimiques et physico-chimiques du muscle n'ont jamais fait l'objet d'études systématiques. Nous avons donc entrepris de préciser par quels mécanismes l'électrochoc peut éventuellement influencer le déroulement de ces phénomènes post mortem.

Dans un premier temps nous avons étudié l'influence de l'électrochoc appliqué à l'animal vivant sur la température et quelques intermédiaires glycolytiques du muscle Longissimus dorsi. Les valeurs obtenues étaient comparées à celles que 1'on observe après l'abattage des animaux, donc après intervention de 1'électrochoc et de la saignée, dont l'effet principal est de rendre le muscle anoxique. Nous avons également vérifié si la réaction à l'électrochoc pouvait expliquer les différences de vitesse de chute du $\mathrm{pH}$ constatées post mortem.

Dans un deuxième temps nous avons étudié l'influence éventuelle de la durée d'application de l'électronarcose sur la vitesse de chute du pH et sur certaines caractéristiques de qualité de la viande qui lui sont étroitement liées.

\section{MATÉRIEL, ET MÉTHODES}

Tous les porcs utilisés au cours de cette étude étaient de race Large White et pesaient i io t $10 \mathrm{~kg}$. Ils étaient achetés dans des élevages privés une semaine avant le début des manipulations.

Influence de l'électronarcose sur quelques caractéristiques physicochimiques du Longissimus dorsi

Vingt et un porcs furent utilisés au cours de cette étude. Toutefois, trois d'entre eux succombèrent à l'application de l'électrochoc et un dut être abattu par suite de fracture. Aussi seuls les résultats obtenus sur les dix-sept animaux restants furent-ils retenus.

Chaque porc était soumis au prélèvement d'une biopsie de muscle Longissimus dorsi, dans la région dorso-lombaire du côté droit, sans anesthésie préalable. Nous avons employé la technique décrite par Schmidt, Zuidam et Sybesma (I97I). Néanmoins les animaux étaient entravés durant l'opération, et recevaient ensuite une injection d'antibiotiques (dihydrostreptomycine : I $\mathrm{g}$; bipénicilline : $\left.10^{6} \mathrm{UI}\right)$

Les échantillons prélevés par biopsie pesaient de Ioo à $200 \mathrm{mg}$. Ils étaient aussitôt plongés dans l'azote liquide, puis rapidement pesés et placés à - $30^{\circ}$. Quelques jours plus tard, ils étaient broyés en présence de $600 \mathrm{mg}$ d'acide perchlorique o,6 $\mathrm{M}$ congelé, dans un mortier refroidi à l'aide d'azote liquide. Le mélange d'acide et de poudre de muscle était transféré dans un récipient de verre préalablement refroidi et maintenu dans la glace jusqu’à décongélation. On ajoutait alors $2,5 \mathrm{ml}$ d'acide perchlorique $0,6 \mathrm{M}$ refroidi à $\mathrm{O}^{\circ} \mathrm{I}^{\circ} \mathrm{C}$ et l'ensemble était homogénéisé grâce à un broyeur Biorex pendant 45 sccondes. Le broyat était centrifugé à $3500 \mathrm{~g}$ pendant io minutes; le surnageant recueilli était neutralisé par la potasse $5 \mathrm{~N}$ en présence de bleu de bromophénol comme indicateur $\left(\mathrm{pH}_{3}, 6-4,2\right)$. Après filtration sur laine de verre pour éliminer le précipité de perchlorate de potassium, le volume final de l'extrait était ajusté à $5 \mathrm{ml}$ avec de l'eau distillée.

Quatre jours plus tard, les animaux recevaient un électrochoc administré grâce à un appareil de marque Etim "polyvalent " destiné à l'abattage industriel des porcs (290 V, 0,4 à o,6 A, 50 périodes). La durée de passage du courant était de une seconde. Pendant ro minutes avant l'éle trochoc et 15 minutes après, la température du muscle Longissimus dorsi droit était continuellement enregistrée grâce à un thermocouple (cuivre-constantan) implanté dans le muscle à une profondeur de $6 \mathrm{~cm}$, au niveau de la région dorso-lombaire et relié à un enregistreur Vitatron. Dès la fin du passage du courant, une biopsie était pratiquée sur le muscle gauche symótriquement à la première. Les animaux recevaient ensuite une nouvelle injection d'antibiotiques.

L'abattage était effectué douze jours après la seconde biopsie, sous électronarcose (courant 
de caractéristiques identiques à celui précédemment décrit). Immédiatement après, puis une heure plus tard, des échantillons de muscle Longissimus dorsi étaient prélevés environ $\mathrm{ro} \mathrm{cm}$ en arrière de la biopsie sur la demi-carcasse gauche. Ils étaient aussitôt plongés dans de l'azote liquide et conservés quelques jours à $-30^{\circ} \mathrm{C}$ avant extraction.

Les échantillons prélevés post mortem étaient broyés, en évitant soigneusement toute décongélation, dans un Waring-Blendor préalablement refroidi à l'aide d'azote liquide. Une partie de la poudre obtenue était extraite dans l'acide perchlorique o,6 M glacé (o- $\left.{ }^{\circ}\right)$ selon la technique décrite par CHARPENTIER (I968). L'autre partie était broyée dans l'iodoacétate $0,005 \mathrm{M}$ refroidi (o à $\left.\mathrm{r}^{\circ} \mathrm{C}\right)(2 \mathrm{~g}$ pour $\mathrm{r} 8 \mathrm{ml}$ de solution) et le $\mathrm{pH}$ déterminé sur le broyat grâce à un pH-mètre Radio meter 29.

Toutes les opérations d'extraction étaient réalisées à une température de o- IoC.

L'ATP, le glucose-6-phosphate, le fructose-I, 6-diphosphate et l'acide lactique ( ${ }^{1}$ ) étaient déterminés dans les extraits perchloriques par les techniques enzymatiques décrites par BERGMEYER (1963) adaptées à l'autoanalyseur Technicon (pour l'ATP la méthode employée utilisait l'hexokinase et la glucose 6-phosphate déshydrogénase).

\section{Infuence de la durée de l'électronarcose}

Le matériel animal utilisé dans cette partie de l'étude comprenait 24 porcs répartis en quatre séries d'abattage de six animaux. Les porcs étaient abattus sous électronarcose, le temps de passage du courant étant fixé à $\mathbf{I}, 2$ ou 3 secondes; chaque traitement était appliqué à deux animaux par série d'abattage, de façon à éliminer l'influence de la date d'abattage sur la vitesse de chute du pH et la qualité de la viande. Toutes les autres opérations (échaudage, éviscérage, fente) étaient conduites de façon identique quel que soit le traitement.

Une heure post mortem un échantillon était prélevé sur le muscle Longissimus dorsi, du côté gauche et dans la région dorso-lombaire. Les prélèvements étaient traités comme décrit précédemment (extraction à l'acide perchlorique et mesure du $\mathrm{pH}$ ). Aussitôt après, les carcasses étaient placées à $+6^{\circ} \mathrm{C}$.

Vingt-quatre heures après l'abattage, la demi-carcasse gauche était découpée et des prélèvements effectués sur les muscles Longissimus dorsi et Biceps femoris. Sur ces échantillons on mesurait le pH grâce à un pH-mètre portatif EIL $30 \mathrm{C}$, l'électrode étant appliquée directement sur la coupe fraîche de muscle. Le pouvoir de rétention d'eau était déterminé par la méthode de Goutefongea (1966) et l'intensité de la coloration à l'aide d'un réflectomètre de CharpentierVerge (Manuflex II).

\section{RÉSULTATS}

\section{I. - Influence de l'électrochoc sur la température et la glycogénolyse musculaire au niveau $d u$ Longissimus dorsi}

Les résultats rapportés dans le tableau I montrent que chez l'animal vivant l'électrochoc entraîne principalement une élévation de la température et du glucose - 6 - phosphate. Les taux d'ATP, de fructose - r,6-diphosphate et d'acide lactique sont également modifiés, mais de façon non significative.

Les variations individuelles de sensibilité à l'électrochoc sont très importantes. Ainsi les augmentations de température enregistrées sont comprises entre o et $\mathrm{I}, 5^{\circ} \mathrm{C}$ selon les animaux. La variabilité des taux de cofacteurs et intermédiaires de la glycolyse déterminés est également beaucoup plus élevée après l'électrochoc qu'au repos.

(1) Dans la suite de notre exposé nous utiliserons les abréviations suivantes :

ATP : adenosine-triphosphate

G-6-P : glucose-6-phosphate

1-1,6-P : fructose-1, 6-diphosphate

$A L$ : acide lactique 
Immédiatement post mortem, à la fin de la saignée, les taux de glucose-6-phosphate, d'ATP et d'acide lactique sont supérieurs à ceux que l'on observe après l'électrochoc chez l'animal vivant. Par contre, le taux de fructose-I, 6-diphosphate est très diminué ; on peut remarquer également qu'il présente, comme in vivo, une grande variabilité.

Les variations du glucose 6-phosphate et de l'acide lactique sont étroitement liées après l'électrochoc in vivo $\left(r=0,83^{* *}\right)$ (tabl. 2). Au contraire les taux d'ATP

\section{TABL,EAU I}

Influence de l'électronarcose

et de la saignée sur quelques caractéristiques du muscle Longissimus dorsi du Porc (moyenne \pm écart type de la moyenne)

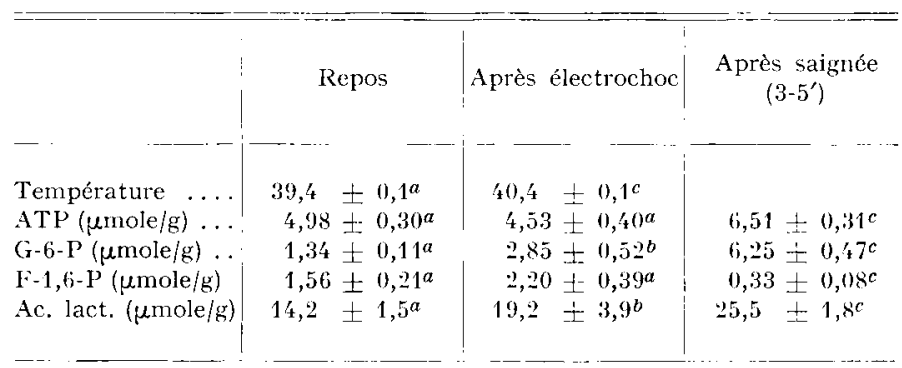

Les valeurs portant une mention difiérente présentent une différence significative : $a-b: \mathrm{P}<0,05 . \quad a-c$ ou $b-c: \mathrm{P}<0,01$.

TABLEAU 2

Corrélation entre différentes caractéristiques du muscle après application de l'électrochoc

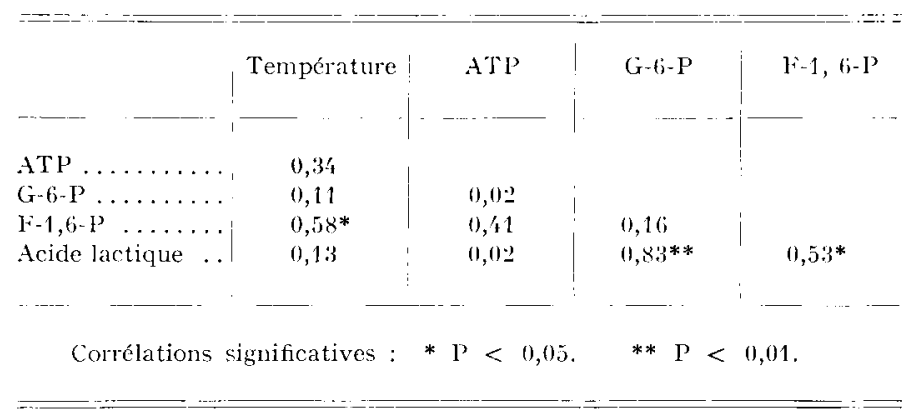

et de fructose-I, 6-diphosphate évoluent relativement indépendamment des deux composés précédents. Il est à noter qu'il y a peu de relations apparentes entre les modifications de la température due à l'électrochoc et les variations des intermédiaires de la glycolyse. 


\section{2. - Relations entre la réaction à l'électrochoc}

et la vitesse de chute du $p H$ post mortem (tabl. 3)

Parmi les caractéristiques mesurées immédiatement après l'électrochoc, la température présente une corrélation assez étroite avec la vitesse de chute du $\mathrm{pH}$. En fait, il semble que la température ultime atteinte par le muscle, influence plus

\section{TABLEAU 3}

Relations entre quelques cavactéristiques mesurées après électrochoc et la chute du pH post mortem dans le muscle Longissimus dorsi du Porc

\begin{tabular}{|c|c|c|c|c|c|c|}
\hline & $\mathrm{Te}$ & Te-Tr & ATP & $\mathrm{G}-6-\mathrm{P}$ & $\mathrm{F}-1,6-\mathrm{P}$ & $A L$ \\
\hline $\mathrm{pH} 0 \mathrm{~h} p . m$ & $-0,53^{*}$ & $-0,48^{*}$ & $-0,22$ & 0,02 & $-0,28$ & $-0,17$ \\
\hline pH 1 h p.m. & $-0,61 * *$ & $-0,46$ & $-0,30$ & $-0,05$ & $-0,41$ & $-0,19$ \\
\hline
\end{tabular}

Corrélations significatives : $* \mathrm{P}<0,05 ; * * \mathrm{P}<0,01$.

Abréviations utilisées :

$\operatorname{Tr}=$ Température au repos.

$\mathrm{Te}=$ Température atteinte après électrochoc.

la glycogénolyse post mortem que l'augmentation proprement due à l'électronarcose.

Par contre, les variations des intermédiaires de la glycolyse consécutives à l'application de la décharge électrique ne semblent pas liées directement à la vitesse de chute du $\mathrm{pH}$.

\section{3. - Influence de la durée de l'électrochoc}

sur la glycogénolyse post mortem et la qualité de la viande

I es résultats rapportés dans le tableau 4 indiquent que la durée d'application de l'électronarcose n'a pas de répercussions sur la glycogénolyse dans le muscle

TABLEAU 4

Infuence de la durée d'application de l'électrochoc sur la glycogénolyse

( $\mathrm{I}$ h post mortem) dans le muscle Longissimus dorsi

(moyenne $t$ écart type de la moyenne)

\begin{tabular}{c|c|c|c|c}
\hline $\begin{array}{c}\text { Durée } \\
\text { (secondes) }\end{array}$ & $\mathrm{pH}$ & $\begin{array}{c}\mathrm{ATP} \\
(\mu \mathrm{mole} / \mathrm{g})\end{array}$ & $\begin{array}{c}\text { G-6-P } \\
(\mu \mathrm{mole} / g)\end{array}$ & $\begin{array}{c}\text { Acide lactique } \\
(\mu \mathrm{mole} / \mathrm{g})\end{array}$ \\
\hline 1 & $6,17 \pm 0,14$ & $5,5 \pm 0,4$ & $3,4 \pm 0,5$ & $48,1 \pm 7,6$ \\
\hline 2 & $\frac{6,12 \pm 0,14}{5,21 \pm 0,12}$ & $5,1 \pm 0,8$ & $3,5 \pm 0,8$ & $46,5 \pm 7,6$ \\
\hline 3 & $\frac{5,7 \pm 0,8}{2,7 \pm 0,4}$ & $50,7 \pm 9,0$ \\
\hline
\end{tabular}


Longissimus dorsi. Aucune différence significative entre les trois traitements n'apparaît en ce qui concerne le $\mathrm{pH}$ ultime, la coloration ou le pouvoir de rétention d'eau (tab1. 5).

\section{TABLEAU 5}

Influence de la durée d'application de l'électrochoc

sur quelques caractéristiques de qualité de la viande, mesurée $24 \mathrm{~h}$ post mortem

\begin{tabular}{c|c|c|c|c|c|c}
\hline \hline \multirow{2}{*}{$\begin{array}{c}\text { Durée } \\
\text { (secondes) }\end{array}$} & \multicolumn{3}{|c|}{ Nuscle Longissimus dorsi } & \multicolumn{3}{|c}{ Muscle Biceps femoris } \\
\cline { 1 - 5 } & $\mathrm{pH}$ & $\% \mathrm{H}_{2} \mathrm{O}$ exp. & Réflectance & $\mathrm{pH}$ & $\% \mathrm{H}_{2} \mathrm{O}$ exp. & Réflectance \\
\hline 1 & $5,64 \pm 0,05$ & $24,2 \pm 2,3$ & $524 \pm 53$ & $5,74 \pm 0,06$ & $23,6 \pm 2,0$ & $405 \pm 46$ \\
\hline 2 & $5,62 \pm 0,04$ & $23,9 \pm 1,4$ & $524 \pm 31$ & $5,76 \pm 0,07$ & $23,1 \pm 2,3$ & $362 \pm 53$ \\
\hline 3 & $5,68 \pm 0,05$ & $21,5 \pm 1,7$ & $500 \pm 10$ & $5,71 \pm 0,06$ & $23,4 \pm 1,7$ & $326 \pm 31$ \\
\hline
\end{tabular}

\section{DISCUSSION}

Les températures que nous avons mesurées au repos dans le muscle Longissimus dorsi sont légèrement plus faibles que celles que rapportent CHARPENTIER, MoniN et Oririvier (I97I), pour la race Large White. Cette différence s'explique sans doute par des modes de manipulation non parfaitement identiques, la température musculaire étant assez facilement modifiée chez le porc par l'excitation ou l'exercice.

Peu de références existent en ce qui concerne les taux des composés intermédiaires de la glycolyse mesurés chez l'animal vivant et conscient. Nous avons toutefois observé des taux de glucose-6-phosphate nettement inférieurs à ceux que rapportent SchuidT, Zuidam et SyBESMa (I97I) : selon ces auteurs le taux de glucose-6-phosphate dans le Longissimus dorsi varie de 1,9 à $7,5 \mu \mathrm{mole} / \mathrm{g}$. Nous avons observé personnellement des valeurs extrêmes au repos de 0,58 à $2,35 \mu$ mole/g. Par contre, en ce qui concerne l'acide lactique, nos résultats sont tout à fait comparables à ceux de ces auteurs.

L'augmentation du taux de glucose-6-phosphate consécutive à l'électrochoc est, quoique significative, très inconstante comme le montre la variabilité très élevée de la valeur obtenue pendant l'électronarcose (tabl. I) ; nous avons observé des différences variant entre $-0,5$ et $+8,6 \mu$ mole $/ g$. Cette accumulation de glucose 6-phosphate peut être attribuée à une activation de la phosphorylase, liée aux contractions musculaires (HELMREICH, KARPATKIn et CORI, I965 ; POSNER, STERN et KREBS, I965), mais aussi à la décharge d'adrénaline qui accompagne l'électrochoc (VAN DER WAL, I97I). Le fait que 1'élévation du taux de glucose-6-phosphate soit très variable n'est pas surprenant; POSNER, STERN et KREBS (Ig65) ont montré en effet que le passage de la phosphorylase $a$ à la phosphorylase $b$, dû à la contraction musculaire, est sujet à des variations individuelles considérables; il en va de même pour la sécrétion d'adrénaline au cours de l'électronarcose comme le montre l'étude des résultats de VAN DER WAL (I97I). 
Les valeurs observées en ce qui concerne la fructose-I, 6-diphosphate sont très variables. Chez certains animaux nous n'avons pu détecter la présence de ce composé en quantité mesurable par nos techniques de dosages. Les taux de F-I,6-P ne paraissent pas liés aux autres intermédiaires de la glycolyse. Il en va de même pour l'A'TP dont les variations au cours de l'électronarcose ne sont pas significatives : ceci semble d'ailleurs logique car les mécanismes de régulation du taux d'ATP, parmi lesquels la glycolyse, sont très efficaces. Par contre, les variations du taux d'acide lactique sont directement liées à celles du G-6-P : ce résultat est en accord avec les travaux de HELMREICH, KARPATKIn et CORI (I965) selon lesquels les contractions musculaires entraînent une élévation concomitante de ces deux composés. La faiblesse de l'augmentation de l'acide lactique comparativement à celle du G-6-P est probablement due en partie à l'évacuation très rapide de ce composé hors du muscle, mais peut-être également au fait que in vivo le pyruvate est métabolisé en majeure partie dans le cycle oxydatif de KREBS.

Lorsque les effets de la saignée se superposent à ceux de l'électrochoc le phénomène le plus remarquable est l'élévation considérable du taux de G-6-P. Il est probable que la décharge d'adrénaline joue alors un rôle supérieur à celui qu'elle occupe au cours de l'électrochoc isolé. D'après HEI Mreich, KARPATKIN et CorI (I965) l'adrénaline augmente en effet la production du G-6-P beaucoup plus rapidement que celle de 1'acide lactique dans le muscle anoxique, contrairement à la contraction musculaire. En outre, la suppression de la circulation sanguine entraîne l'accumulation de l'acide lactique, dont le taux devient significativement supérieur à celui que l'on observait après l'électrochoc seul. Dans l'ensemble nos résultats sont en accord avec ceux de ChARPENTIER (I968), compte tenu de la vitesse de chute du $\mathrm{pH}$ post mortem ( $\mathrm{pH}$ I $\mathrm{h}$ post mortem $6,3 \mathrm{I} \pm 0,20$ dans notre étude), sauf en ce qui concerne le F-I, 6-P pour lequel CharPENTIER observait des valeurs nettement supérieures (environ $3 \mu \mathrm{M} / \mathrm{g}$ ).

Notons, au sujet de ce dernier composé, que les valeurs rapportées par différents auteurs, à vitesse de chute de $\mathrm{pH}$ équivalente, sont extrêmement variables. Ainsi Kastenschmidt, Hoekstra et Briskey (I966) rapportent des taux de $6 \mu$ moles/g immédiatement post mortem dans le Longissimus dorsi de porcs présentant une chute de $\mathrm{pH}$ lente $(\mathrm{pH}$ I $\mathrm{h}$ post mortem $\geqslant 6,0)$. La signification des variations du taux de F-r, 6-P vis-à-vis de la vitesse de glycogénolyse ne semble pas connue. On pourrait attribuer ces variations à des différences dans l'activité des enzymes glycolytiques responsables de sa formation (phospho-fructokinase) ou de sa dégradation (aldolase), mais par ailleurs l'activité de ces enzymes ne semble jouer aucun rôle dans le déterminisme de la vitesse de chute du pH (BENDALL, I965).

L'augmentation de température observée après l'électrochoc (parfois jusqu'à $\left.\mathrm{I}, 5^{\circ} \mathrm{C}\right)$ ne semble pas devoir être attribuée uniquement à la tétanisation de la musculature. En effet chez certains animaux cette élévation se poursuit plusieurs minutes après la fin du passage du courant, alors que les muscles paraissent relâchés. Il est possible que la décharge d'adrénaline soit responsable pour une part de cet effet de l'électronarcose sur la température musculaire. L'existence d'une corrélation assez étroite $\left(0,6 I^{* *}\right)$ entre la température après électrochoc et la vitesse de chute $\mathrm{du} \mathrm{pH}$ s'explique d'après les travaux de BENDALL, HALLUND et WISMER-PEDERSEN (I963). Ces auteurs ont montré qu'une augmentation de température relativement faible peut accélérer sensiblement la glycogénolyse post mortem chez le porc, le 
coefficient de température $\left(Q_{10}\right)$ de la chute du $\mathrm{pH}$ entre $36^{\circ}$ et $4 \mathrm{I}^{\circ}$ atteignant 2,70. Or après électrochoc nous avons observé des températures musculaires de $39,3^{\circ} \mathrm{C}$ à $4 \mathrm{I}, 5^{\circ} \mathrm{C}$. La température agit sur la glycogénolyse post mortem en favorisant l'altération du réticulum sarcoplasmique due à la chute du pH musculaire (CHARPENTIER, I97I). Il est donc logique que les animaux qui présentent après électrochoc la température musculaire la plus élevée manifestent post mortem la chute de $\mathrm{pH}$ la plus rapide, compte tenu du fait que l'interruption de la circulation sanguine diminue les possibilités d'évacuation des calories au niveau du muscle et que les convulsions au cours de la saignée ne peuvent qu'aggraver cet état de chose.

Il est, par contre, surprenant que l'allongement de la durée d'application de l'électrochoc n'entraîne aucune modification de la chute du $\mathrm{pH}$ musculaire post mortem, et par suite des caractéristiques de qualité de la viande qui lui sont liées. Il semble donc bien que l'intensité de la réaction, au niveau du muscle, au passage du courant dans les centres nerveux soit liée à des différences individuelles de sensibilité entre animaux plutôt qu'à la durée de stimulation : nous avons en effet observé une proportion comparable de pores présentant une chute de $\mathrm{pH}$ rapide dans les trois groupes.

Comme nous l'avons noté dans l'exposé “ Matériel et Méthodes », l'électrochoc a provoqué chez trois animaux des fractures accompagnées d'hémorragies importantes au niveau de la ceinture pelvienne (à l'insertion du fémur sur le coxal). Deux de ces porcs ont succombé très rapidement, mais le troisième ne présentait par ailleurs aucun signe clinique. Ces fractures peuvent être attribuées à la contracture musculaire qui accompagne le passage du courant dans les centres nerveux. $\mathrm{Au}$ cours de nombreux abattages réalisés avec le même appareillage, mais les pores étant suspendus par un membre postérieur avant l'application de l'électronarcose, nous n'avons jamais constaté de tels incidents. Cette influence de la position des porcs lors de l'abattage mériterait d'être étudiée de manière plus approfondie car les fractures et les hémorragies peuvent justifier la saisie de parties de la carcasse par les services d'Inspection sanitaire, et sont donc à l'origine de pertes économiques.

\section{CONCL,USION}

Ėn définitive il apparaît que, parmi les composantes de la réaction à l'électronarcose utilisée pour l'abattage des porcs, l'élévation de température musculaire a une influence fondamentale sur la vitesse de chute du $\mathrm{pH}$ post mortem. Vis-à-vis de la rapidité de la glycogénolyse musculaire et, par suite, de la qualité de la viande, les différences de sensibilité individuelles des animaux à l'électrochoc semblent plus importantes que la durée d'application de celui-ci. A cause de sa facilité, la mesure de la température musculaire pourrait se révéler particulièrement utile dans la mise au point des techniques d'abattage et l'étude de leur influence sur la qualité de la viande de porc. Toutefois des études ultérieures se révèlent nécessaires pour préciser les mécanismes d'action de l'électronarcose au niveau musculaire et les causes des différences individuelles de sensibilité des animaux. 


\section{SUMMARY}

\section{ELECTRICAL, STUNNING AND POST MORTEM GLYCOGENOL,YSIS IN THE PIG.}

It has been well established that the method used for Pig stunning before slaughter affects the post mortem glycogenolytic rate in the muscles. The aim of this study was to determine how electrical stunning can give raise to muscular $\mathrm{pH}$ fall after death.

Temperature and some glycolytic intermediates (ATP, G-6-P, F-I, 6-P, lactic acid) were determined in the Longissimus dorsi muscle of $\mathbf{I} 7$ pigs before and after electroshock stunning, and immediately after slaughtering. Temperature raise and G-6-P accumulation are observed after electroshock. It seems that temperature raise plays the most important role in accelerating the post mortem glycolytic rate.

Glycogenolysis and meat quality are not influenced by electroshock duration. Thus, it seems that $\mathrm{pH}$ fall is related to individual differences of susceptibility to electroshock. The mechanisms of muscle reaction to electroshock must be explained in further experiments.

\section{RÉFÉRENCES BIBLIOGRAPHIQUES}

BENDALL R., I965. The effect of pre-treatment of pigs with curare on the post mortem rate of $\mathrm{pH}$ fall and onset of rigor mortis in the musculature. XIth European Meeting of Meat Research Workers. August 1965, Belgrade.

Bendall R., Hallund O., Wismer-Pedersen J., 1963. Post mortem changes in the muscles of Landrace pigs. J. Food Sci., 28, I56-I62.

Bergmeyer H. U., I963. Methods of enzymatic analysis. Academic Press, New York.

Charpentier J., i 968 . Cilycogénolyse post mortem du muscle Iongissimus dorsi de porc. Ann. Zootech., 17, $429-443$.

Charpenter J., i97i. Caractéristiques biochimiques du réticulum sarcoplasmique du muscle de porc post mortem. Ann. Biol. anim. Bioch. Biophys., 11, 427-434.

Charpentier J., Monin G., Ollivier L., i97i. Relations entre les réactions du porc à un choc thermique et la qualité de la viande. Journées d'études de la Commission de Génétique de la F. E. Z., I7I 9 juillet I97I, Versailles.

Goutefongea R., I966. Eitude comparative de différentes méthodes de mesure du pouvoir de rétention d'eau de la viande de porc. Ann. Zootech., 15, 291-295.

Helmreici E., Karpation S., Cori C. li., I965. Regulation of glycolysis in skeletal muscle. In control of glycogen metabolism C. I. B. A. Foundation Symposium. Churchill lutd Editors, London.

Kastinschmidt L. L., Hofkstra W. G., 13кiskey E. J., ig66. Metabolic intermediates in skeletal muscles with fast and slow rates of post mortem glycolysis. Nature, 2 I 2-288.

McLovghlin, I97. The death reaction and metabolism post mortem of porcine skeletal muscle. General introduction, Section 2, Stunning. In Proceedings of the Second Int. Symp. on Condition and Meat Quality of pigs. March 22-24, 1971, Zeist, the Netherlands.

Mclovghlin J. V., Tarrant P. S. V., ig68. Post mortem changes in muscle taken from live pigs and from pigs immediately after slaughter. Int. Symposium on Meat Quality. May 6-1o r968. Zeist. The Netherlands.

MONin G., I97I. Influence d'une injection de sulfate de magnésium sur le déroulement de la glycogénolyse post mortem dans le muscle de porc. Ann. Zootech., 20, 497-507.

Posver J. B., Stern R., Krebs F. G., I965. Effects of electrical stimulation and epinephrine on muscle phosphorylase, phosphorylase $b$-kinase and adenosin $3^{\prime}, 5^{\prime}$-phosphate. J. Biol. Chem., 240, $9^{82-985}$.

Ratcliff P. W., I971. Review of papers, Section 2, Stunning. In. Proceedings of the Second Int. Symp. on Condition and Meat Quality of pigs. March 22-24, I97r. Zeist, the Netherlands.

Sair R. A., Lister D., Moody W. C., Cassens R. G., Hoekstra W. C., Briskey E. J., ig7o. Action of curare and magnesium on striated muscle of stress susceptible pigs. Am. J. Physiol., 218 , IO8-II4.

Schmidt G. R., Zuidam L., Sybesma W., i97i. Biopsy technique and analysis for predicting pork quality. In Proceedings of the Second Int. Symp. on Condition and Meat Quality of pigs. March 22-24, I971. Zeist, the Netherlands.

VAN DER WaL P. G., I97I. Stunning, sticking and exsanguination as stress factors in pigs. In Proceedings of the Second Int. Symp. on Condition and Meat Quality of pigs. March 22-24, I97I. Zeist, the Netherlands. 